\title{
Chemical Functionalization of Graphene Nanoribbons
}

\author{
Narjes Gorjizadeh and Yoshiyuki Kawazoe \\ Institute for Materials Research, Tohoku University, Sendai 980-8577, Japan \\ Correspondence should be addressed to Narjes Gorjizadeh, narjes@imr.edu
}

Received 31 May 2010; Accepted 28 June 2010

Academic Editor: Rakesh Joshi

Copyright ( 2010 N. Gorjizadeh and Y. Kawazoe. This is an open access article distributed under the Creative Commons Attribution License, which permits unrestricted use, distribution, and reproduction in any medium, provided the original work is properly cited.

\begin{abstract}
We review the electronic properties of graphene nanoribbons functionalized by various elements and functional groups. Graphene nanoribbons are strips of graphene, the honeycomb lattice of carbon with $s p^{2}$ hybridization. Basically nanoribbons can be classified into two categories, according to the geometry of their edge, armchair, and zigzag, which determine their electronic structure. Due to their fascinating electronic and magnetic properties many applications has been suggested for these materials. One of the major methods to use graphene nanoribbons in future applications is chemical functionalization of these materials to make an engineering on their band gap. In this review, we introduce various types of modifying graphene nanoribbons to meet their promising applications.
\end{abstract}

\section{Introduction}

After discovery of graphene [1], it has been considered as basic material for the future nanoelectronic devices. Its unique properties, such as massless Dirac fermion behavior [2-4], half-integer quantum Hall effect $[2,5]$, and highcarrier mobility [2] make it a promising candidate for application in nanoelectronics and spintronics devices $[2,5-$ 9]. Graphene nanoribbons can be constructed as strips of graphene, with quasilD structures. So far, Nanoribbons with widths up to $2 \mathrm{~nm}$ have been fabricated experimentally [1012]. Geometrically, two main types of nanoribbons with two edge shapes can be cut from a hexagonal lattice of graphene: zigzag edge and armchair edge [13-16]. Different types of ribbons are specified by their edge geometry and width. The width is labeled by an integer which counts the number of carbon chains between the two edges. Figure 1 shows the two types of ribbons with their width indices. The two ribbon characteristics, that is, edge geometry and width, are the key parameters which determine the electronic properties of the ribbons [7, 17-19].

The earliest theoretical studies of graphene nanoribbons, using a simple tight-binding method, predicted that $1 / 3$ of the armchair nanoribbons, whose width index $N$ satisfies $N=3 M-1$ ( $M$ is an integer), are metallic [17], and another
$2 / 3$ are semiconductor with band gaps depending on their width, while all zigzag nanoribbons are metallic, a similar behavior as carbon nanotubes (CNTs). A characteristic peak in the density of states (DOS) of zigzag nanoribbons near and slightly below Fermi energy is also predicted $[16,20]$. But recently the first principle studies based on spin polarized density functional theory (DFT) revealed that all graphene nanoribbons are semiconductors at their ground state with band gaps which depend on their width and edge geometry, closing at infinite width, that is, infinite graphene $[7,21]$. Meanwhile zigzag nanoribbons have localized edge states which are ferromagnetically ordered, but with opposite spin orientation at the two edges which makes them antiferromagnetically coupled. The magnetism in zigzag nanoribbon, a pure carbon system, which arises from $\pi$-orbitals of carbon localized at the edge is specially notable [22]. These properties, along with the ballistic electronic transport, and quantum Hall effect [2,5], cause graphene nanoribbons to be promising candidates for building blocks of future nanoelectronic and spintronic devices $[1,2,5-7,23-30]$ and also chemical sensors [31-33] and electrochemical switches [34].

In order to achieve their potential for these applications it is essential to have a better understanding of the electronic structure of graphene nanoribbons and have ability 


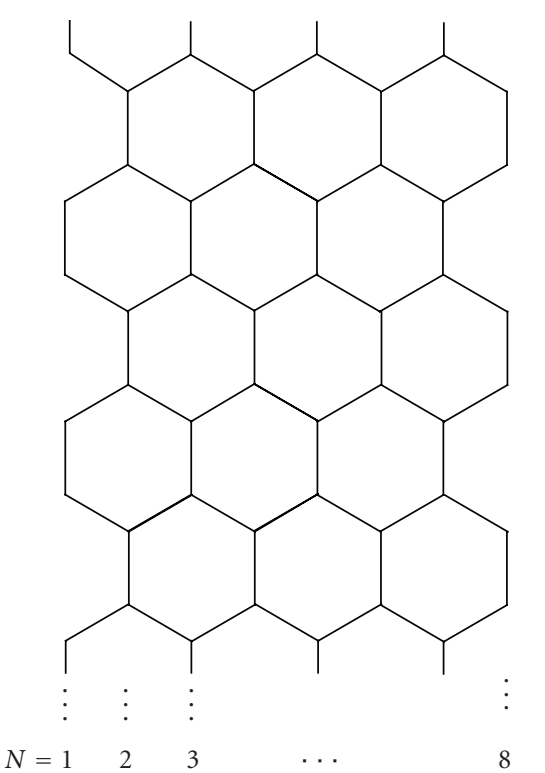

(a)

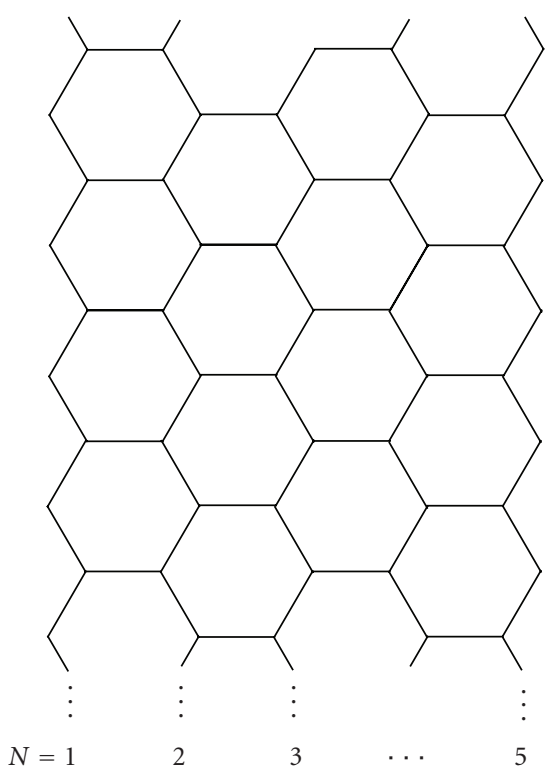

(b)

FIGURE 1: Graphene nanoribbons with armchair (a) and zigzag (b) edges. In each case, index $N$ denotes the width of the ribbon, and ribbon axis is the vertical direction.

to control them. Modification of electronic structure of nanoribbons by chemical functionalization is an effective way to make them efficient for their applications [35-39].

Moreover, various junctions can be constructed by connecting nanoribbons of different widths and types with perfect atomic interface, and electronic device can be integrated on them by selective chemical functionalization on a single nanoribbon sheet [38, 40]. Yan et al. [41] illustrated that nanoribbon-based devices can be made with the atomic-perfect-interface junctions and with controlled doping through edge termination.
So far many theoretical studies have been done to functionalize graphene nanoribbons to manipulate their electronic properties. Here we review different ways of chemical functionalization of nanoribbons and their properties.

\section{Functionalization of Graphene Nanoribbons}

Electronic structure of graphene nanoribbons can be modified by several types of functionalization, such as functionalization by s-type, p-type, and d-type elements [38, 42$44]$, or by functional groups. Modification of the edge of the ribbon, or substitution of one or more carbons of the ribbon or adsorption of one atom or a functional group are different options of functionalization which have been all investigated theoretically and some of them has been observed experimentally.

2.1. Edge Modification. Nanoribbons intrinsically have dangling bonds at the edges whose linear combinations form some of the eigenstates near the Fermi energy, and hence determine the ribbon properties. These dangling bonds also provide active sites for chemical bonding, making the ribbons suitable for chemical modification. Since the unique properties of the ribbons are associated with their edge states, edge modification can significantly affect and control electronic and magnetic properties of the ribbons [37, 45, 46]. By functionalization of the edges with various atoms, from $s$-type to $d$-type transition metals, and by functional groups, we can obtain various electronic properties. In fact, the same ribbon can become semiconductors with wide range of band gaps, metallic, ferromagnetic (FM) and antiferromagnetic (AF) or half-metallic by appropriate chemical functionalization. Experimentally, the edge dangling bonds of the graphene sheets and ribbons can be saturated by hydrogen through specific hydrogenation procedures.

The first step in modification of nanoribbon is to saturate one of the edges by an element or functional group while the other edge is saturated by $\mathrm{H}$. It was demonstrated by theoretical calculations that different functional groups at the edge of nanoribbons can significantly affect their electronic structure close to Fermi level [37]. Decoration of one edge of zigzag nanoribbons by two $\mathrm{H}$ atoms while the other has one $\mathrm{H}$ turns the antiferromagnetic nanoribbon into ferromagnetic [47]. Single-edge functionalization of zigzag ribbons leads to half-semiconductors with different band gaps for each spin and can also result in a spin-polarized half-semiconductor or in a semiconductor-metal transition [48]. Modification of one or both edges of zigzag nanoribbon with functional groups $-\mathrm{O},-\mathrm{F},-\mathrm{OH},-\mathrm{NH}_{2},-\mathrm{CH},-\mathrm{BH}$, and -B was also investigated [35, 37, 39, 45]. Figure 2 depicts schematic picture of a zigzag nanoribbon with three types of edge saturation.

In zigzag nanoribbons the atoms of one edge are ferromagnetically ordered, but the two edges are antiferromagnetically coupled, and the band structure of the two spin channels are degenerate. Applying an electric field across a zigzag ribbon results in lifting the spin degeneracy by reducing the band gap for one spin channel while widening 


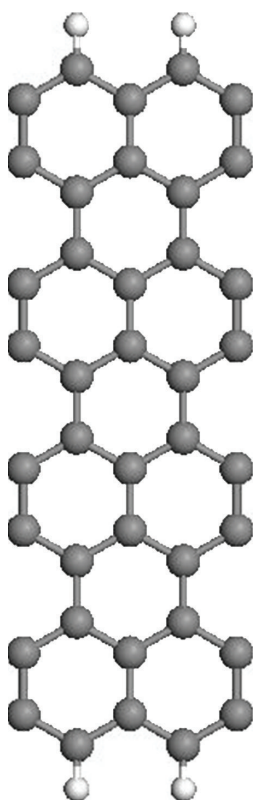

(a)

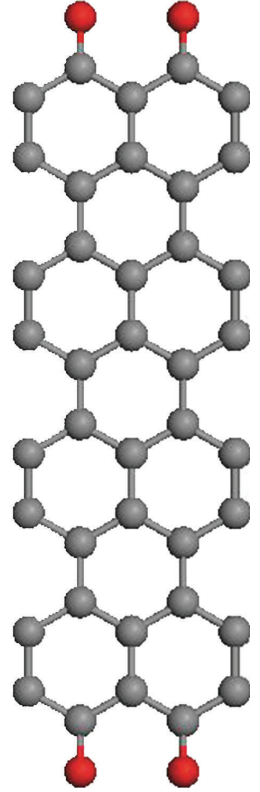

(b)

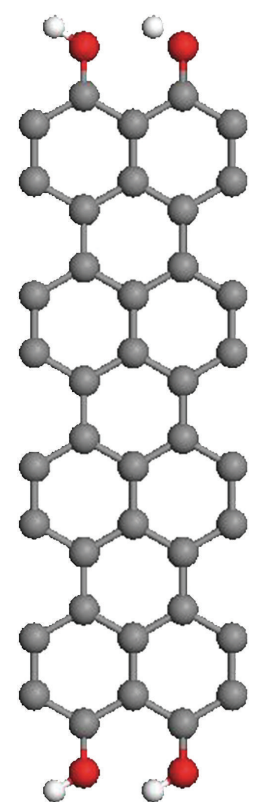

(c)
Figure 2: Zigzag nanoribbon with edge saturation by (a) $\mathrm{H}$ (b) $\mathrm{O}$ and (c) $\mathrm{OH}$. Gray atoms are $\mathrm{C}$, white atoms are $\mathrm{H}$ and red atoms are O.

the gap of the other spin channel $[6,7]$, which makes the ribbon half-metallic [49]. In half-metals one spin channel has metallic behavior, while the other one has energy band gap. This phenomena provides a spin selective behavior for nanoribbons which is a promise for spintronics applications. It was theoretically predicted that in these nanoribbons edge oxidization can have significant effect in designing efficient and robust spintronic devices based on nanoribbons [45]. Nanoribbons with fully oxidized edges turn half-metallic at low-electric field $(0.2 \mathrm{~V} / \AA)$, while for fully or partially hydrogenated ribbons the filed intensity needed to switch the system to half metallic regime is $(0.4 \mathrm{~V} / \AA)$ [ 45$]$.

Besides electric field, the degeneracy of spin channels can be broken by chemical modifications also. Modifying one edge of the nanoribbon with electron donating groups and the other edge with electron accepting groups is a sufficient way to generate large potential difference between the two edges [44]. This way the corresponding potential shift of band structure of spin up and spin down are different and the system may become half metallic. Kan et al. [44] have shown that replacing the terminating $\mathrm{H}$ atoms of one edge of zigzag ribbon by $\mathrm{NO}_{2}$ groups and the other edge with $\mathrm{CH}_{3}$ groups turns the ribbon into half-metal. Wu et al. [42] also suggested that half-metallicity can be realized in zigzag nanoribbons while one edge is modified with $\mathrm{OH}$ and the other edge is decorated with $\mathrm{NO}_{2}$ or $\mathrm{SO}_{2}$.

Chemical modification of armchair nanoribbons is also effective to engineer their band gaps. Saturation of the edge of armchair nanoribbons by $\mathrm{B}$ attachment as a bridge between edge carbon atoms in the planar direction (Figure 3(b)) results in metallic behavior of the system, while $\mathrm{N}$ attached at the same geometry widens the gap

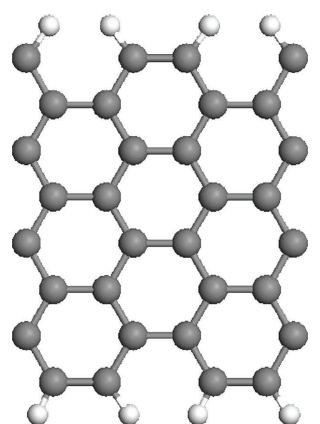

(a)

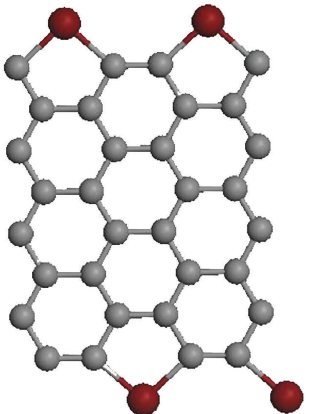

(b)
FIgURE 3: Armchair nanoribbon with edge saturation by (a) $\mathrm{H}$ and (b) B.

of the ribbon [38]. However, substitution of both edges by $\mathrm{B}$ or $\mathrm{N}$ keeps the ribbon substantially unchanged [48], while full half-metallicity can be achieved by substitution of one edge carbon by $\mathrm{B}$ atoms and the other edge by $\mathrm{N}$ atoms [50]. Decorating the edge of armchair nanoribbons, which are nonmagnetic semiconductors, in the position of Figure 3(b) by transition metals provides ferromagnetic or antiferromagnetic states depending on the type of the decorated atom [38]. However, edge functionalization of armchair ribbon by functional group $\mathrm{NH}_{2}$ does not alter the electronic properties of the nanoribbon since the impurity states are located far away from the Fermi level [48].

\subsection{Substitution. Substitution of one or more carbons of} the nanoribbon by introducing impurities to the system is another effective way to control the electronic properties of the nanoribbons. Intrinsically $\mathrm{B}$ and $\mathrm{N}$ impurities play the role of p-type and n-type doping in carbon-based materials. But in nanoribbons the doping process is extremely sensitive to the dopant position and density, type and width of the ribbon $[40,51,52]$. If $\mathrm{B}(\mathrm{N})$ substitution occurs in the center of the armchair ribbons, the defect states appear close to the bottom (top) of the conduction (valance) band of the pristine ribbon, but the Fermi energy is shifted in the conduction (valance) band, resulting in a semiconductormetal transition, rather than doping [48]. However by B and $\mathrm{N}$ substitution at the ribbon edges, the impurity levels are always very far from the Fermi energy and the electronic properties of the ribbon are substantially unchanged [48], while substitution of edge atoms in zigzag nanoribbons induce semiconductor-metal transition for high impurity densities, but do not give impurity levels close to the top or bottom of the gap [48].

Zheng et al. [50] have shown that zigzag nanoribbons can be either semiconducting, half metallic, or metallic by controlling the distance of the $\mathrm{N}$ or B impurity atoms to the edge. In these ribbons half metallicity can be achieved by substitution of one edge carbon with $\mathrm{B}$ atoms and the other edge with $\mathrm{N}$ atoms [50]. Half-metallicity can be also achieved by replacing middle zigzag chains of a zigzag nanoribbon by B-N chains (Figure 4) [53]. Replacing periodically the 


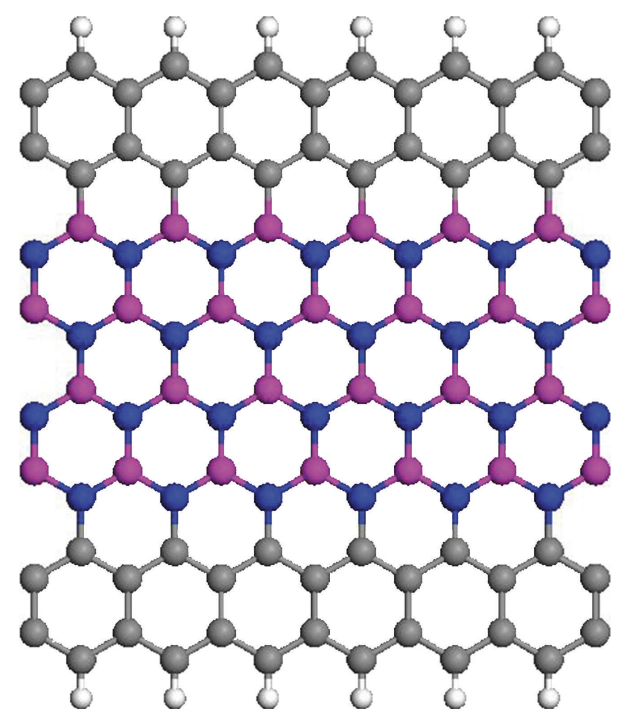

Figure 4: Hydrogen saturated zigzag nanoribbon. The middle zigzag chains of carbon are substituted by B-N chains.

armchair chains across the zigzag nanoribbon by B-N chain will also result in half metallicity [54].

2.3. Adsorption. Substitution of $\mathrm{N}$ and $\mathrm{B}$ has be shown to have significant effect on the electronic structure of nanoribbons. However controlling the doping site and density is a key factor in achieving the desired properties. Adsorption of additional atoms or functional groups is another effective way to modify the electronic structure of nanoribbons. Yu et al. [55] have shown that the properties of armchair nanoribbons can be strongly modified by adsorption of $\mathrm{N}$ atoms at the edge. First principle calculations of B, C, and $\mathrm{N}$ adsorbed atoms on zigzag nanoribbons have also revealed that these adatoms convert the antiferromagnetic ribbon into ferromagnetic with high magnetic moment [46]. These adatom prefer the sites near the edge of the ribbon. On the other hand, $\mathrm{NO}_{2}$ and $\mathrm{NH}_{2}$ which act as strong acceptors and donors on graphene surface can modify the electronic structure of zigzag nanoribbons in the gap vicinity when adsorbed at the edge, but do not act as a dopant [48].

However, adsorption of transition metals on armchair nanoribbons turns the nonmagnetic ribbon into a magnetic metal [56]. Depending on the width of the ribbon and type of adsorbed transition metals, armchair nanoribbon can either become a metal or a semiconductor with ferromagnetic or antiferromagnetic spin alignment. Fe or Ti adsorption makes certain armchair nanoribbons half-metallic [56]. Adsorption of $\mathrm{Ni}$ atom on zigzag nanoribbons was also investigated [57].The most stable position of the adsorbed $\mathrm{Ni}$ is at the edge site, which reduces the magnetic moments of both $\mathrm{Ni}$ atom and the edge $\mathrm{C}$ due to hybridization of $d$ orbitals of Ni with $p$ orbitals of C [57]. First principle studies of adsorption of $\mathrm{Ni}_{n}$ and $\mathrm{Fe}_{n}$ clusters $(n=1-4)$ on zigzag nanoribbons shows that clusters also prefer the edge sites, and the system is more stable in antiferromagentic coupling of the zigzag edges [57].
Zigzag nanoribbons can also become half-metallic by deposition of the ferroelectric material polyvinylidene flouride (PVDF) on them [43]. PVDFs generate the electrostatic potential on the nanoribbon because of their strong dipole moments. The dipole direction of PVDFs is changeable by an external electric field, and switching between half-metallic and insulating states in nanoribbon may be acheived [43].

The possibility of chemical doping and related chemical sensor properties of graphene have been demonstrated experimentally. $\mathrm{NO}_{2}$ molecule is found to be a strong acceptor in experimental observations [32].

\section{Conductance}

Understanding the effect of chemical functionalization and edge topology on charge transport in graphene junctions is an important issue. So far various theoretical studies of the influence of edge geometry and chemical modifications on the transport properties of nanoribbons have been reported [48]. Simulations of electronic transport of realistic edge disordered armchair nanoribbons predicts that transport properties strongly depend on the geometry of the reconstructed edge profile and local defect chemical reactivity [58].

Quantum transport calculations of B doped armchair nanoribbons by substitution of carbons with $\mathrm{B}$ by various doping rates indicates that depending on the energy of the charge carrier, the transport can vary from quasiballistic to a strongly localized regime, as a result of strong electron-hole asymmetry induced by chemical doping [34]. The occurrence of quasibound states related to B impurities results in mobility gaps as large as $1 \mathrm{eV}$, due to strong electronhole asymmetrical backscattering phenomena, which opens new ways to overcome current limitations of graphenebased devices through the fabrication of chemically doped graphene nanoribbons [34].

Martins et al. [59] also show that substitutional B atoms at the ribbon edge acts as scattering centers for the electronic transport along the ribbon which breaks the symmetry of spin-up and spin-down channels. Transport of one of the spin channels is reduced more strongly, which gives rise to spin-polarized transport by suitable doping [59].

First principle calculations show that presence of $\mathrm{B}$ and $\mathrm{N}$ impurities in armchair nanoribbons results in resonant backscattering, whose features are strongly dependent on the symmetry and the width of nanoribbon, as well as the position and dopants [51]. Energies of the quasibound states are strongly dependent on the position of the impurity with respect to the edges. When $B$ is placed at the exact center of the ribbon the transmission at the first plateau is insensitive to the presence of the impurity, while B impurity at the edge reduces the conductance due to the quasibound states induced by the impurity [51].

Ab-initio calculations of conductance in armchair nanoribbons with joint attachment of $\mathrm{OH} / \mathrm{H}$ and phenyl groups on their surface also shows that transport properties depend on the adsorption sites [60]. If $\mathrm{OH}$ bond is located at the ribbon edge, conductance is not affected. The same result is obtained for $\mathrm{NH}_{2}$ adsorption at the edge of armchair 
ribbons [48]. But when the $\mathrm{OH}$ bond is shifted out of the edge, the conductance is typically disturbed, and a rapid decay in conductance is obtained by increasing the defect density [60]. Dependence of Conductance on the defect site and its distance from the edge was also reported by [61]

Conductance measurements of nanoribbons has been also experimentally afforded [62]. All these studies opens the way to manipulate graphene nanoribbon-based devices and development of new applications based on functionalized nanoribbons.

\section{Summary and Perspectives}

Graphene nanoribbon has a great potential to be used in the future electronic applications. Working on desirable properties by modifying nanoribbons by appropriate elements or functional groups helps us find the suitable form of graphene nanoribbon for each application. Modification with various types of elements and functional groups can give us a variety of properties, such as semiconducting with a wide range of band gap, metallic, ferromagnetic, antiferromagnetic, half-metallic, half-semiconducting, all obtained from the same nanoribbon. Modification of the edge or using an adsorbate or substitution of carbons of the nanoribbon with an appropriate host are different options to meet the desired property from the nanoribbon. Applicability of each option would be proven by experiments.

\section{Acknowledgments}

The authors would like to express their sincere thanks to the crew of the Center for Computational Materials Science, Institute for Materials Research, for their support of the Hitachi SR1 1000 (model K2) supercomputer system and also Global COE program of Tohoku University for financial support.

\section{References}

[1] K. S. Novoselov, A. K. Geim, S. V. Morozov, et al., "Electric field in atomically thin carbon films," Science, vol. 306, no. 5696, pp. 666-669, 2004.

[2] K. S. Novoselov, A. K. Geim, S. V. Morozov et al., "Twodimensional gas of massless Dirac fermions in graphene," Nature, vol. 438, no. 7065, pp. 197-200, 2005.

[3] M. I. Katsnelson, K. S. Novoselov, and A. K. Geim, "Chiral tunnelling and the Klein paradox in graphene," Nature Physics, vol. 2, no. 9, pp. 620-625, 2006.

[4] A. K. Geim and K. S. Novoselov, "The rise of graphene," Nature Materials, vol. 6, no. 3, pp. 183-191, 2007.

[5] Y. Zhang, Y.-W. Tan, H. L. Stormer, and P. Kim, "Experimental observation of the quantum Hall effect and Berry's phase in graphene," Nature, vol. 438, no. 7065, pp. 201-204, 2005.

[6] Y.-W. Son, M. L. Cohen, and S. G. Louie, "Half-metallic graphene nanoribbons," Nature, vol. 444, no. 7117, pp. 347349, 2006.

[7] Y.-W. Son, M. L. Cohen, and S. G. Louie, "Energy gaps in graphene nanoribbons," Physical Review Letters, vol. 97, no. 21, Article ID 216803, 4 pages, 2006.

[8] B. Obradovic, R. Kotlyar, F. Heinz, et al., "Analysis of graphene nanoribbons as a channel material for field-effect transistors,"
Applied Physics Letters, vol. 88, no. 14, Article ID 142102, 3 pages, 2006.

[9] Z. Li, H. Qian, J. Wu, B.-L. Gu, and W. Duan, "Role of symmetry in the transport properties of graphene nanoribbons under bias," Physical Review Letters, vol. 100, no. 20, Article ID 206802, 4 pages, 2008.

[10] M. C. Lemme, T. J. Echtermeyer, M. Baus, and H. Kurz, "A graphene field-effect device," IEEE Electron Device Letters, vol. 28, no. 4, pp. 282-284, 2007.

[11] X. Li, X. Wang, L. Zhang, S. Lee, and H. Dai, "Chemically derived, ultrasmooth graphene nanoribbon semiconductors," Science, vol. 319, no. 5867, pp. 1229-1232, 2008.

[12] P. Gallagher, K. Todd, and D. Goldhaber-Gordon, "Disorderinduced gap behavior in graphene nanoribbons," Physical Review B, vol. 81, no. 11, Article ID 115409, 8 pages, 2010.

[13] Y. Niimi, T. Matsui, H. Kambara, K. Tagami, M. Tsukada, and H. Fukuyama, "Scanning tunneling microscopy and spectroscopy of the electronic local density of states of graphite surfaces near monoatomic step edges," Physical Review B, vol. 73, no. 8, Article ID 085421, 8 pages, 2006.

[14] Y. Kobayashi, K.-I. Fukui, T. Enoki, K. Kusakabe, and Y. Kaburagi, "Observation of zigzag and armchair edges of graphite using scanning tunneling microscopy and spectroscopy," Physical Review B, vol. 71, no. 19, Article ID 193406, 4 pages, 2005.

[15] F. Sols, F. Guinea, and A. H. C. Neto, "Coulomb blockade in graphene nanoribbons," Physical Review Letters, vol. 99, no. 16, Article ID 166803, 4 pages, 2007.

[16] Y. Niimi, T. Matsui, H. Kambara, K. Tagami, M. Tsukada, and H. Fukuyama, "Scanning tunneling microscopy and spectroscopy studies of graphite edges," Applied Surface Science, vol. 241, no. 1-2, pp. 43-48, 2005.

[17] M. Fujita, K. Wakabayashi, K. Nakada, and K. Kusakabe, "Peculiar localized state at zigzag graphite edge," Journal of the Physical Society of Japan, vol. 65, no. 7, pp. 1920-1923, 1996.

[18] R. Saito, M. Fujita, G. Dresselhaus, and M. S. Dresselhaus, "Electronic structure of chiral graphene tubules," Applied Physics Letters, vol. 60, no. 18, pp. 2204-2206, 1992.

[19] D. J. Klein, "Graphitic polymer strips with edge states," Chemical Physics Letters, vol. 217, no. 3, pp. 261-265, 1994.

[20] K. Sasaki, S. Murakami, and R. Saito, "Stabilization mechanism of edge states in graphene," Applied Physics Letters, vol. 88, no. 11, Article ID 113110, 3 pages, 2006.

[21] L. Pisani, J. A. Chan, B. Montanari, and N. M. Harrison, "Electronic structure and magnetic properties of graphitic ribbons," Physical Review B, vol. 75, no. 6, Article ID 064418, 9 pages, 2007.

[22] K. Wakabayashi, M. Fujita, H. Ajiki, and M. Sigrist, "Electronic and magnetic properties of nanographite ribbons," Physical Review B, vol. 59, no. 12, pp. 8271-8282, 1999.

[23] Y. Zhang, Z. Jiang, J. P. Small, et al., "Landau-level splitting in graphene in high magnetic fields," Physical Review Letters, vol. 96, no. 13, Article ID 136806, 4 pages, 2006.

[24] O. Hod and G. E. Scuseria, "Electromechanical properties of suspended graphene nanoribbons," Nano Letters, vol. 9, no. 7, pp. 2619-2622, 2009.

[25] X. Lü, Y. Zheng, H. Xin, and L. Jiang, "Spin polarized electron transport through a graphene nanojunction," Applied Physics Letters, vol. 96, no. 13, Article ID 132108, 3 pages, 2010.

[26] L. Zhu, J. Wang, T. Zhang, et al., "Mechanically robust tri-wing graphene nanoribbons with tunable electronic and magnetic properties," Nano Letters, vol. 10, no. 2, pp. 494-498, 2010. 
[27] Y. Yang and R. Murali, "Impact of size effect on graphene nanoribbon transport," IEEE Electron Device Letters, vol. 31, no. 3, pp. 237-239, 2010.

[28] T. Ozaki, K. Nishio, H. Weng, and H. Kino, "Dual spin filter effect in a zigzag graphene nanoribbon," Physical Review B, vol. 81, no. 7, Article ID 075422, 5 pages, 2010.

[29] K. Pi, W. Han, K. M. McCreary, A. G. Swartz, Y. Li, and R. K. Kawakami, "Manipulation of spin transport in graphene by surface chemical doping," Physical Review Letters, vol. 104, no. 18, Article ID 187201, 4 pages, 2010.

[30] O. V. Yazyev and M. I. Katsnelson, "Magnetic correlations at graphene edges: basis for novel spintronics devices," Physical Review Letters, vol. 100, no. 4, Article ID 047209, 4 pages, 2008.

[31] F. Schedin, A. K. Geim, S. V. Morozov et al., "Detection of individual gas molecules adsorbed on graphene," Nature Materials, vol. 6, no. 9, pp. 652-655, 2007.

[32] T. O. Wehling, K. S. Novoselov, S. V. Morozov et al., "Molecular doping of graphene," Nano Letters, vol. 8, no. 1, pp. 173-177, 2008.

[33] S. Alwarappan, A. Erdem, C. Liu, and C.-Z. Li, "Probing the electrochemical properties of graphene nanosheets for biosensing applications," Journal of Physical Chemistry C, vol. 113, no. 20, pp. 8853-8857, 2009.

[34] B. Biel, F. Triozon, X. Blase, and S. Roche, "Chemically induced mobility gaps in graphene nanoribbons: a route for upscaling device performances," Nano Letters, vol. 9, no. 7, pp. 27252729, 2009.

[35] M. Maruyama, K. Kusakabe, S. Tsuneyuki, K. Akagi, Y. Yoshimoto, and J. Yamauchi, "Magnetic properties of nanographite with modified zigzag edges," Journal of Physics and Chemistry of Solids, vol. 65, no. 2-3, pp. 119-122, 2004.

[36] M. Maruyama and K. Kusakabe, "Theoretical prediction of synthesis methods to create magnetic nanographite," Journal of the Physical Society of Japan, vol. 73, no. 3, pp. 656-663, 2004.

[37] D. Gunlycke, J. Li, J. W. Mintmire, and C. T. White, "Altering low-bias transport in zigzag-edge graphene nanostrips with edge chemistry," Applied Physics Letters, vol. 91, no. 11, Article ID 112108, 3 pages, 2007.

[38] N. Gorjizadeh, A. A. Farajian, K. Esfarjani, and Y. Kawazoe, "Spin and band-gap engineering in doped graphene nanoribbons," Physical Review B, vol. 78, no. 15, Article ID 155427, 6 pages, 2008.

[39] M. H. Wu, Y. Pei, and X. C. Zeng, "Planar tetracoordinate carbon strips in edge decorated graphene nanoribbon," Journal of the American Chemical Society, vol. 132, no. 16, pp. 5554-5555, 2010.

[40] B. Huang, Q. Yan, G. Zhou et al., "Making a field effect transistor on a single graphene nanoribbon by selective doping," Applied Physics Letters, vol. 91, no. 25, Article ID 253122, 3 pages, 2007.

[41] Q. Yan, B. Huang, J. Yu, et al., "Intrinsic current-voltage characteristics of graphene nanoribbon transistors and effect of edge doping," Nano Letters, vol. 7, no. 6, pp. 1469-1473, 2007.

[42] M. H. Wu, X. Wu, and X. C. Zeng, "Exploration of half metallicity in edge-modified graphene nanoribbons," Journal of Physical Chemistry C, vol. 114, no. 9, pp. 3937-3944, 2010.

[43] Y.-L. Lee, S. Kim, C. Park, J. Ihm, and Y.-W. Son, "Controlling half-metallicity of graphene nanoribbons by using a ferroelectric polymer," ACS Nano, vol. 4, no. 3, pp. 1345-1350, 2010.

[44] E.-J. Kan, Z. Li, J. Yang, and J. G. Hou, "Half-metallicity in edge-modified zigzag graphene nanoribbons," Journal of the
American Chemical Society, vol. 130, no. 13, pp. 4224-4225, 2008.

[45] O. Hod, V. Barone, J. E Peralta, and G. E. Suseria, "Enhanced half-metallicity in edgeoxidized zigzag graphnene nanoribbons," Nano Letters, vol. 7, no. 8, pp. 2295-2299, 2007.

[46] E. Kan, H. Xiang, F. Wu, C. Lee, J. Yang, and M.-H. Whangbo, "Ferrimagnetism in zigzag graphene nanoribbons induced by main-group adatoms," Applied Physics Letters, vol. 96, no. 10, Article ID 102503, 3 pages, 2010.

[47] B. Xu, J. Yin, Y. D. Xia, X. G. Wan, K. Jiang, and Z. G. Liu, "Electronic and magnetic properties of zigzag graphene nanoribbon with one edge saturated," Applied Physics Letters, vol. 96, no. 16, Article ID 163102, 3 pages, 2010.

[48] F. Cervantes-Sodi, G. Csányi, S. Piscanec, and A. C. Ferrari, "Edge-functionalized and substitutionally doped graphene nanoribbons: electronic and spin properties," Physical Review B, vol. 77, no. 16, Article ID 165427, 13 pages, 2008.

[49] J.-H. Park, E. Vescovo, H.-J. Kim, C. Kwon, R. Ramesh, and T. Venkatesan, "Direct evidence for a half-metallic ferromagnet," Nature, vol. 392, no. 6678, pp. 794-796, 1998.

[50] X. H. Zheng, X. L. Wang, T. A. Abtew, and Z. Zeng, "Building half-metallicity in graphene nanoribbons by direct control over edge states occupation," Journal of Physical Chemistry C, vol. 114, no. 9, pp. 4190-4193, 2010.

[51] B. Biel, X. Blase, F. Triozon, and S. Roche, "Anomalous doping effects on charge transport in graphene nanoribbons," Physical Review Letters, vol. 102, no. 9, Article ID 096803, 4 pages, 2009.

[52] S. S. Yu, W. T. Zheng, Q. B. Wen, and Q. Jiang, "First principle calculations of the electronic properties of nitrogen-doped carbon nanoribbons with zigzag edges," Carbon, vol. 46, no. 3, pp. 537-543, 2008.

[53] S. Dutta, A. K. Manna, and S. K. Pati, "Intrinsic halfmetallicity in modified graphene nanoribbons," Physical Review Letters, vol. 102, no. 9, Article ID 096601, 4 pages, 2009.

[54] E.-J. Kan, X. Wu, Z. Li, X. C. Zeng, J. Yang, and J. G. Hou, "Half-metallicity in hybrid BCN nanoribbons," Journal of Chemical Physics, vol. 129, no. 8, Article ID 084712, 5 pages, 2008.

[55] S. S. Yu, W. T. Zheng, and Q. Jiang, "Electronic properties of nitrogen-atom-adsorbed graphene nanoribbons with armchair edges," IEEE Transactions on Nanotechnology, vol. 9, no. 2, pp. 243-247, 2010.

[56] H. Sevinçli, M. Topsakal, E. Durgun, and S. Ciraci, "Electronic and magnetic properties of $3 d$ transition-metal atom adsorbed graphene and graphene nanoribbons," Physical Review B, vol. 77, no. 19, Article ID 195434, 7 pages, 2008.

[57] V. A. Rigo, T. B. Martins, A. J. R. da Silva, A. Fazzio, and R. H. Miwa, "Electronic, structural, and transport properties of Ni-doped graphene nanoribbons," Physical Review B, vol. 79, no. 7, Article ID 075435, 9 pages, 2009.

[58] S. M. M. Dubois, A. López-Bezanilla, A. Cresti et al., "Quantum transport in graphene nanoribbons: effect of edge reconstruction and chemical reactivity," ACS Nano, vol. 4, no. 4, pp. 1971-1976, 2010.

[59] T. B. Martins, R. H. Miwa, A. J. R. da Silva, and A. Fazzio, "Electronic and transport properties of boron-doped graphene nanoribbons," Physical Review Letters, vol. 98, no. 19, Article ID 196803, 4 pages, 2007.

[60] A. López-Bezanilla, F. Triozon, and S. Roche, "Chemical functionalization effects on armchair graphene nanoribbon transport," Nano Letters, vol. 9, no. 7, pp. 2537-2541, 2009. 
[61] N. Gorjizadeh, A. A. Farajian, and Y. Kawazoe, "The effects of defects on the conductance of graphene nanoribbons," Nanotechnology, vol. 20, no. 1, Article ID 015201, 6 pages, 2009.

[62] M. Y. Han, B. Özyilmaz, Y. Zhang, and P. Kim, "Energy bandgap engineering of graphene nanoribbons," Physical Review Letters, vol. 98, no. 20, Article ID 206805, 4 pages, 2007. 

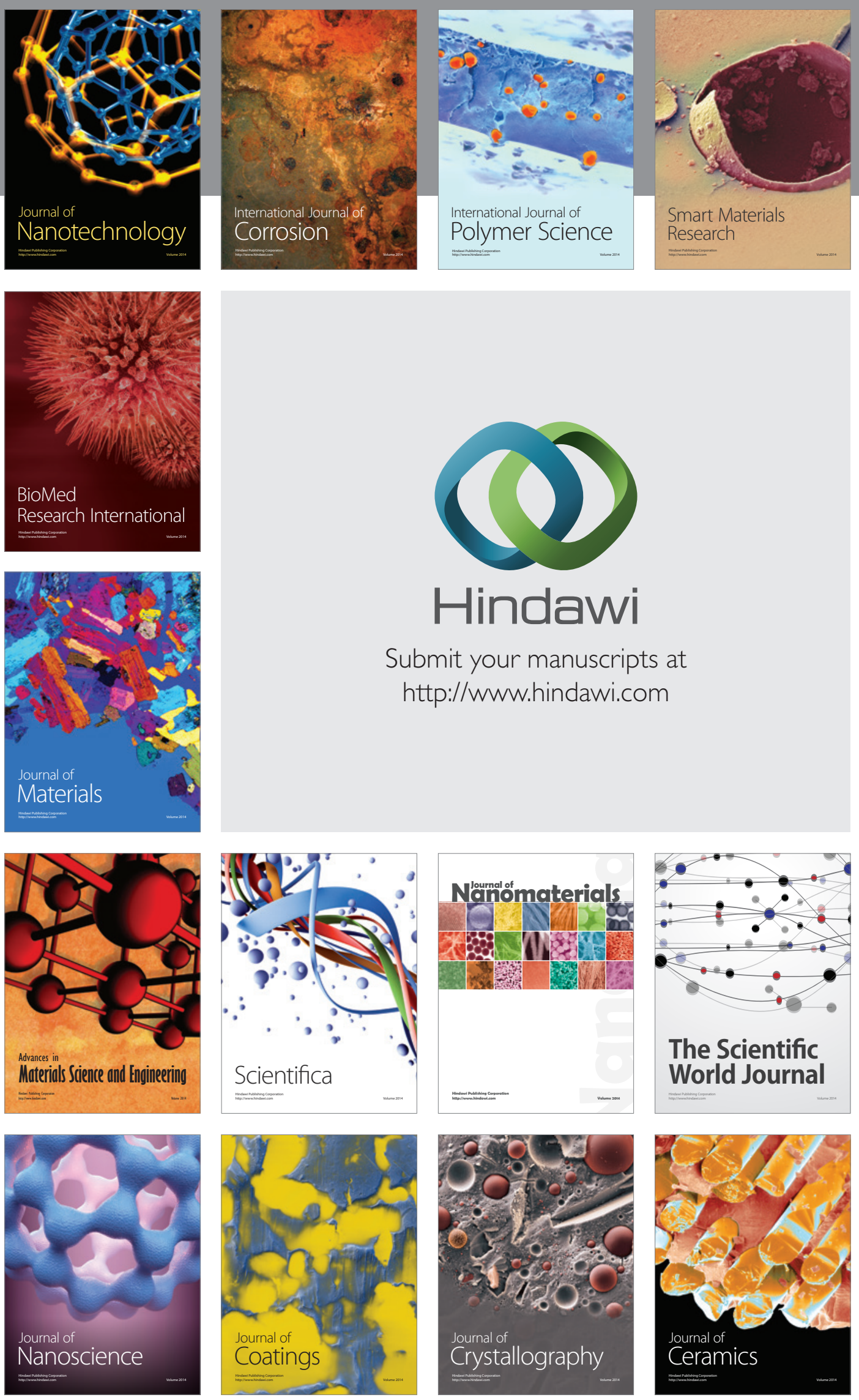

The Scientific World Journal

Submit your manuscripts at

http://www.hindawi.com

\section{World Journal}

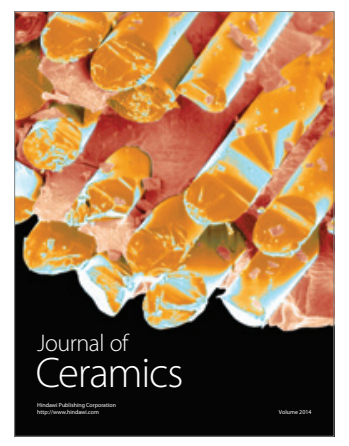

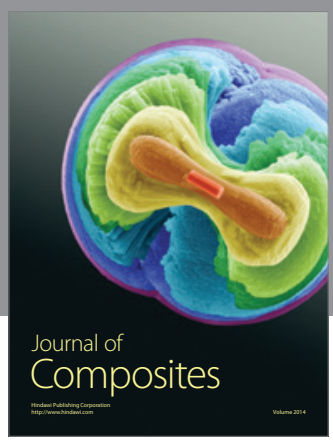
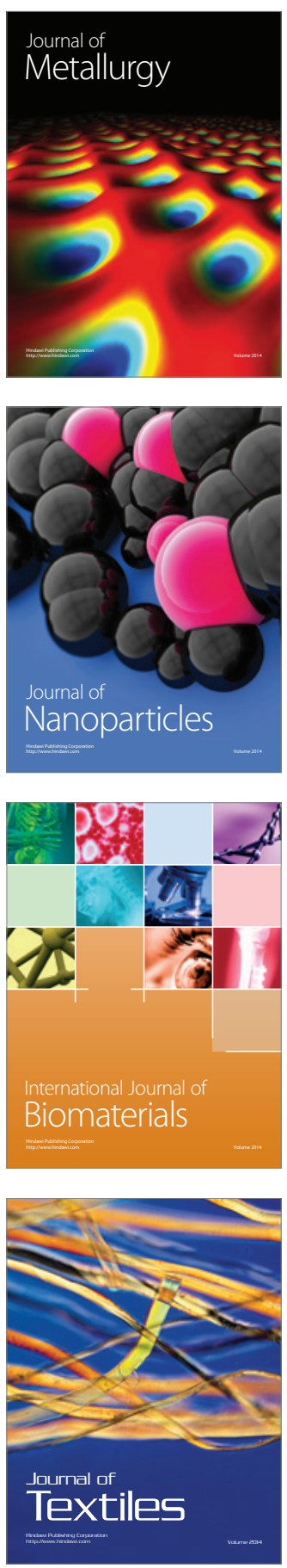